\title{
Fourth-Order Cumulants to Characterize the Phase Transitions of a Spin-1 Ising Model
}

\author{
Shan-Ho Tsai \\ Institute for Theoretical Physics \\ State University of New York \\ Stony Brook, N. Y. 11794-3840, USA \\ and S. R. Salinas \\ Instituto de Física \\ Universidade de São Paulo \\ Caixa Postal 66318 \\ 05315-970, São Paulo, SP, Brazil
}

Received October 6, 1997

\begin{abstract}
Fourth-order cumulants of physical quantities have been used to characterize the nature of a phase transition. In this paper we report some Monte Carlo simulations to illustrate the behavior of fourth-order cumulants of magnetization and energy across second and firstorder transitions in the phase diagram of a well known spin-1 Ising model. Simple ideas from the theory of thermodynamic fluctuations are used to account for the behavior of these cumulants.
\end{abstract}

\section{Introduction}

There are many attempts to characterize the order of a phase transition on the basis of the analysis of numerical data obtained from simulations of finite spin systems. One of the approaches to this problem consists in the analysis of the behavior of fourth-order cumulants of physical quantities (as the order parameter and the energy) associated with the systems under consideration[1, 2]. Properties of the fourth-order cumulants of magnetization (and energy) have been investigated in the context of finite-size effects in magnetically (and thermally) driven first-order transitions in Ising and Potts models[3, 4, 5, 6] (as well as in the case of some other systems[7, 8]).

In this paper, we perform Monte Carlo simulations for the well known Blume-Capel model[9, 10] to illustrate the behavior of the fourth-order cumulants of magnetization and energy across first and second-order transitions in the phase diagram of this system. We show that it is possible to draw some conclusions from the study of relatively small lattices. The general features of the cumulants can be accounted for by simple arguments from the theory of thermodynamic fluctuations. In particular, we emphasize the differences between the two types of cumulants, and the alternative definitions of the cumulant of energy (which has not been fully appreciated in previous investigations).

The layout of this paper is as follows. In Section 2 we define the cumulants of a physical quantity. In Section 3 we introduce some further definitions, and discuss some properties of the Blume-Capel model. Simulations for the fourth-order cumulants of magnetization and energy are reported in Sections 4 and 5, respectively. We hope to have provided another example of the use of these cumulants to characterize the order of a phase transition.

\section{Definition of the cumulants}

The cumulants of a quantity $x$ can be obtained from an expansion of the form 


$$
<\exp (x)>=1+<x>+\frac{1}{2}<x^{2}>+\frac{1}{6}<x^{3}>+\frac{1}{24}<x^{4}>+\ldots,
$$

where the brackets denote an average[11]. If we keep terms up to fourth order, the logarithm of this expansion may be written as

$$
\ln \{<\exp (x)>\}=<x>+\frac{1}{2} Q_{2}+\frac{1}{6} Q_{3}+\frac{1}{24} Q_{4}+\ldots,
$$

where the cumulants $Q_{2}, Q_{3}$, and $Q_{4}$, are given by

$$
\begin{gathered}
Q_{2}=<x^{2}>-<x>^{2}, \\
Q_{3}=<x^{3}>-3<x><x^{2}>+2<x>^{3},
\end{gathered}
$$

and

$$
Q_{4}=\left\langle x^{4}>-3<x^{2}>^{2}-4<x><x^{3}>+12<x>^{2}<x^{2}>-6<x>^{4} .\right.
$$

These cumulants can be rewritten in the more compact form,

$$
\begin{aligned}
& Q_{2}=<(x-<x>)^{2}>, \\
& Q_{3}=<(x-<x>)^{3}>,
\end{aligned}
$$

and

$$
Q_{4}=<(x-<x>)^{4}>-3<(x-<x>)^{2}>^{2} .
$$

Also, the fourth-order cumulant $Q_{4}$ is more often written as

$$
V_{x}(L)=1-\frac{<\left(x-<x>_{L}\right)^{4}>_{L}}{3<\left(x-<x>_{L}\right)^{2}>_{L}^{2}}
$$

where $L$ is the linear size of the lattice under consideration.

\section{The Blume-Capel model}

The Blume-Capel model is given by the spin Hamiltonian

$$
\mathcal{H}=-J \sum_{(i, j)} S_{i} S_{j}+D \sum_{i=1}^{N} S_{i}^{2}-H \sum_{i=1}^{N} S_{i},
$$

where $S_{i}=+1,0,-1$, on sites $i=1, \ldots, N$ of a Bravais lattice, and the first sum is performed over all pairs of nearest-neighbor sites. We consider the ferromagnetic case, with positive exchange $(J>0)$ and anisotropy $(D>0)$ parameters, which gives rise to a competition between distinct spin orderings. In the $D / J$ versus $T / J$ space, where $T$ is the absolute temperature, the phase diagram consists of an ordered and a disordered phase separated by a transition line that changes character from first to second-order at a well defined tricritical point. We use this model to illustrate the behavior of the fourth-order cumulants.

In zero field, the ordered phase is characterized by symmetric magnetizations, $+m_{0}$ and $-m_{0}$, with the same energy. In general, we have $\langle\mathcal{H}\rangle_{L} \neq 0$, and even $\left\langle\mathcal{H}^{n}\right\rangle_{L} \neq 0$, for all $n$. Therefore, the fourth-order cumulant of energy is written as

$$
V_{E}(L)=1-\frac{<\left(\mathcal{H}-<\mathcal{H}>_{L}\right)^{4}>_{L}}{3<\left(\mathcal{H}-<\mathcal{H}>_{L}\right)^{2}>_{L}^{2}}
$$

where $L$ is the linear size of the lattice. As the magnetization is symmetric, that is, $\left\langle m^{n}\right\rangle_{L}=0$ for $n$ odd, the fourth-order cumulant of the magnetization is given by

$$
V_{m}(L)=1-\frac{<m^{4}>_{L}}{3<m^{2}>_{L}^{2}} .
$$

It should be remarked that, as $\left\langle E^{n}\right\rangle_{L} \neq 0$ for all $n$ (including odd values of $n$ ), and $<m^{n}>_{L}=0$ for odd $n$, the energy and the magnetization give rise to distinct expressions of the fourth-order cumulant.

Now we perform a preliminary Monte Carlo simulation to look at the form of the distribution of probabilities for the magnetization (and to motivate the choice of the distributions of energy and magnetization to be used in the forthcoming theoretical calculations). Let 
us define the dimensionless variables

$$
t \equiv \frac{T}{J}, \quad d \equiv \frac{D}{J}, \text { and } \quad h \equiv \frac{H}{J},
$$

and use a Metropolis algorithm to perform Monte Carlo simulations for the Blume-Capel model, in zero field, on a simple cubic lattice of side $L$.
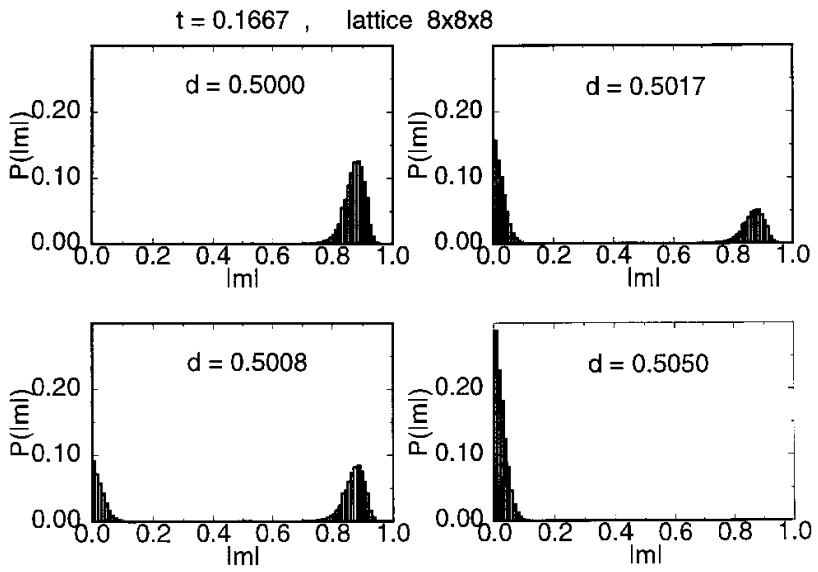

Figure 1. Histogram of the absolute values of the magnetization, $|m|$, across a first-order phase transition.

In Fig. (1), we show the distribution of the absolute values of the magnetization for $t=0.1667, L=8$, and several values of $d$. In the top left graph, for $d=0.5000$, $|m|$ has a unique maximum around $|m|=0.9$, which corresponds to an ordered phase. The bottom left graph, for $d=0.5008$, displays two peaks (around $m=0$ and $|m|=0.9$ ), which indicate the coexistence of phases $\left(m=0\right.$, and $m= \pm m_{0} \neq 0$ ). Upon increasing the value of $d$, the peak at $|m|=0$ is enhanced, while the peak around $|m|=0.9$ is depressed (see the top right graph, for $d=0.5017$ ). For even larger values of $d$, there remains a single peak around $|m|=0$, which indicates that the system is in the paramagnetic phase (see the graph for $d=0.5050$ ). This set of graphs represents a first-order transition.
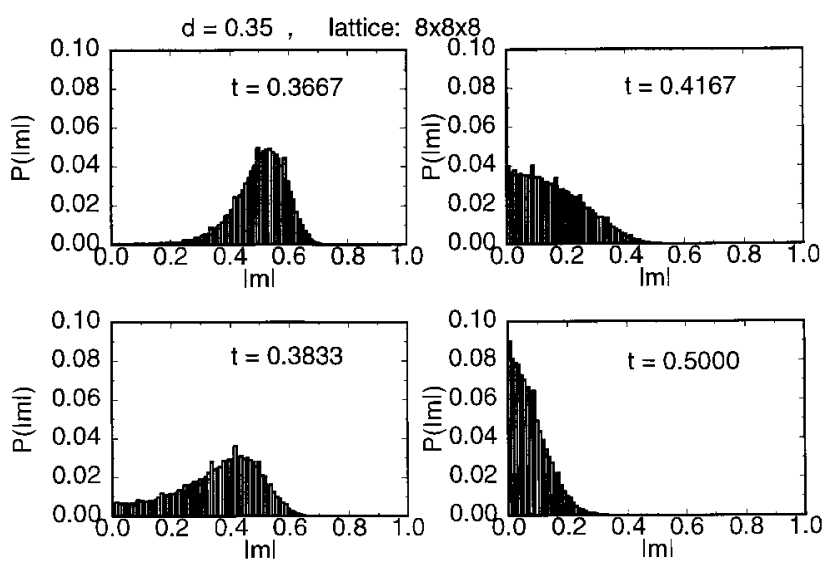

Figure 2. Histogram of the absolute values of the magnetization, $|m|$, across a second-order phase transition.

In Fig. (2), we show a histogram of $|m|$ across a second-order transition, for $d=0.35$. The top left graph, for $t=0.3667$, corresponds to the ordered phase, with a single peak of the absolute value of the magnetization at $|m|=0.5$. As the value of $t$ is increased, this peak moves toward $|m|=0$ and no other peak arises (see the lower left graph, for $t=0.3833$ ). For higher values of $t,|m|$ peaks at $|m|=0$ (see the graphs at right, for $t=0.4167$ and $t=0.5000)$. This set of graphs illustrates a continuous phase transition.

Figs. (1) and (2) provide the motivation for choosing a Gaussian form for the probability of magnetization, $p(m)$. Although we are showing data for the magnetization, similar histograms can be built for the energy, which also give support to a Gaussianshaped probability distribution, $p(E)$. For large lattices $(L \rightarrow \infty)$ these distributions are expected to tend to Dirac delta functions. The thermodynamic consistency of these assumptions has been discussed in detail by Challa, Landau, and Binder[4] (see also the work of Oitmaa and Fernandez[12]).

Now we present separate analyses of the fourthorder cumulants associated with magnetization and energy across first and second-order phase transitions.

\section{Cumulants of magnetization}

In the ordered phase the distribution of probability of the magnetization consists of two peaks around $+m_{0}$ and $-m_{0}$. For small lattices ( $L$ finite), we assume the Gaussian form 


$$
p(m)=\frac{1}{2} C \exp \left[-\frac{\left(m+m_{o}\right)^{2}}{2 \sigma^{2}}\right]+\frac{1}{2} C \exp \left[-\frac{\left(m-m_{o}\right)^{2}}{2 \sigma^{2}}\right],
$$

where $C=\left(2 \pi \sigma^{2}\right)^{-1 / 2}$ is a normalization constant, and the parameter $\sigma$ should be inversely proportional to the lattice volume, $L^{3}$. In the $L \rightarrow \infty$ limit, we have two Dirac delta functions,

$$
p(m)-\frac{1}{2} \delta\left(m+m_{o}\right)+\frac{1}{2} \delta\left(m-m_{o}\right) .
$$

For finite lattices, with $p(m)$ given by Eq. (14), we have $\left\langle m^{n}\right\rangle=0$, for odd values of $n$, and

$$
<m^{2}>=\int_{-\infty}^{\infty} m^{2} p(m) d m=\sigma^{2}+m_{o}^{2}
$$

and

$$
<m^{4}>=\int_{-\infty}^{\infty} m^{4} p(m) d m=3 \sigma^{4}+6 \sigma^{2} m_{o}^{2}+m_{o}^{4}
$$

Thus, the fourth-order cumulant is given by

$$
V_{m}(L)=1-\frac{3 \sigma^{4}+6 \sigma^{2} m_{o}^{2}+m_{o}^{4}}{3\left(\sigma^{2}+m_{o}^{2}\right)^{2}}
$$

In the disordered phase, $p(m)$ has a single peak at $m_{0}=0$. Inserting $m_{0}=0$ in Eq. (18), we have $V_{m}(L)=0$ for the disordered phase.

For infinite lattices $(\sigma \rightarrow 0)$, it is easy to see that $V_{m}(L) \rightarrow 2 / 3$ in the ordered phase. In the disordered phase, $V_{m}(L) \rightarrow 0$, regardless of the value of the parameter $\sigma$. These limiting values for the infinite lattice also come from the double-delta distribution $p(m)$, given by Eq. (15). In this case, in the ordered phase, we have $<m^{n}>=0$, for odd $n$, and $<m^{n}>=m_{o}^{n}$ for even $n$. Thus

$$
V_{m}(L) \rightarrow 1-\frac{m_{o}^{4}}{3\left(m_{o}^{2}\right)^{2}}=\frac{2}{3}
$$

In the disordered phase, $\left\langle m^{n}\right\rangle=0$ for all $n$, hence $V_{m}(L) \rightarrow 0$.

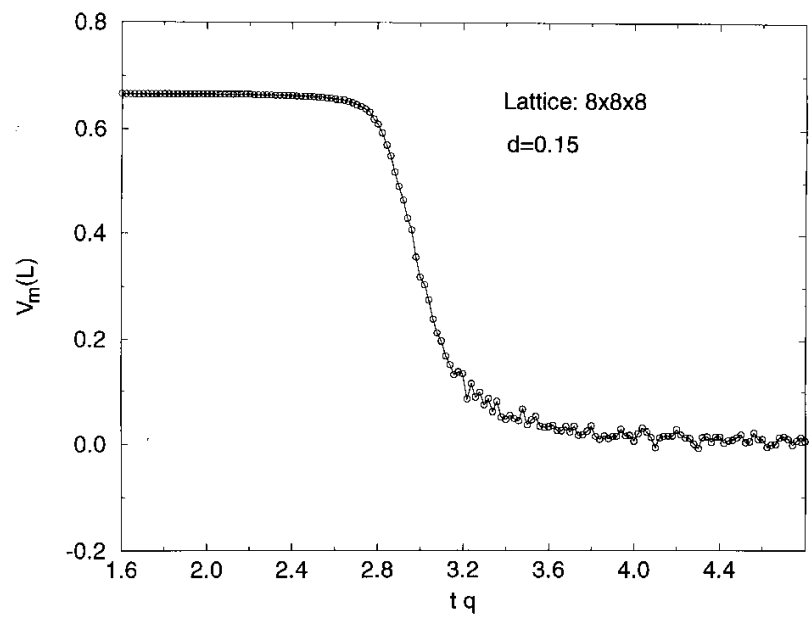

Figure 3. Fourth-order cumulant of the magnetization versus temperature, $t q$, across a second-order phase transition. Monte Carlo data were obtained for the Blume-Capel model on a cubic lattice $(q=6)$ of side $L=8$, for $d=0.15$. Averages were calculated from 50000 Monte Carlo steps after thermalization.

In a second order phase transition, the two peaks of the distribution of probabilities $p(m)$ in the ordered phase move towards each other and form a unique peak at $m=0$ as the system passes to the disordered phase. Fig. (3) shows the cumulant of magnetization for the Blume-Capel model in a second order phase transition. These simulations were performed for a cubic lattice of side $L=8$ (and coordination $q=6$ ), at $d=0.15$. We have run 5000 times through the lattice to reach thermalization. Each average was then calculated using 50000 additional steps.

In a first-order phase transition we have the coexistence of the ordered and disordered phases. The distribution of probabilities $p(m)$ has peaks at $m= \pm m_{0} \neq$ 0 , and $m=0$. For an infinite lattice, we take the tripledelta distribution,

$$
p(m)=c \delta\left(m+m_{o}\right)+c \delta\left(m-m_{o}\right)+(1-2 c) \delta(m),
$$

where $c$ is a positive constant. We then have

$$
<m^{2}>=\int_{-\infty}^{\infty} m^{2} p(m) d m=2 c m_{o}^{2}
$$


and

$$
<m^{4}>=\int_{-\infty}^{\infty} m^{4} p(m) d m=2 c m_{o}^{4} .
$$

Therefore,

$$
V_{m}(L)=1-\frac{2 c m_{o}^{4}}{3\left(2 c m_{o}^{2}\right)^{2}}=1-\frac{1}{6 c} .
$$

For small values of $c$, namely $c<1 / 6$, the cumulant $V_{m}(L)$ is negative. Similar results could have been obtained from the $L \rightarrow \infty$ limit of a Gaussian form of $p(m)$ for a finite lattice. In Fig. (4), we show the fourthorder cumulant of the magnetization across a first-order phase transition. The simulations were performed for a cubic lattice, with $L=8$, at $t=0.15$. We have run 5000 steps through the lattice to reach thermalization. Each average was then calculated from 50000 additional steps. In the ordered phase, we do have $V_{m}(L)=2 / 3$. In the disordered phase there are very small fluctuations around $V_{m}(L)=0$. At the transition, $V_{m}(L)$ assumes a negative value.

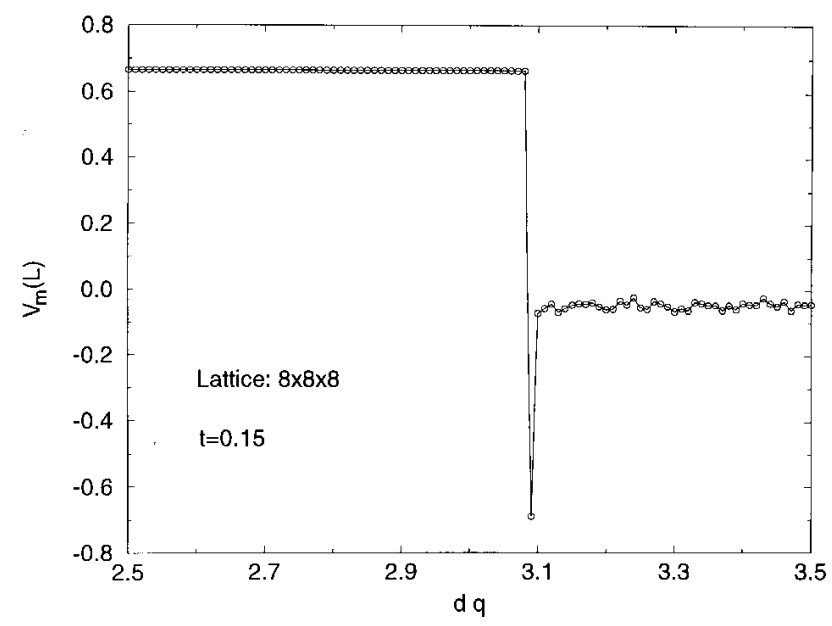

Figure 4. Fourth-order cumulant of magnetization versus anisotropy, $d q$, across a first-order phase transition. Monte Carlo data were obtained for the Blume-Capel model on a cubic lattice $(q=6)$ of side $L=8$, at temperature $t=0.15$. Averages were calculated from 50000 Monte Carlo steps after thermalization.

\section{Cumulants of energy}

In both ordered and disordered phases, the probability distribution of energy has a unique peak at a certain value, which we call $E_{0}$. For small lattices, we can write the Gaussian form

$$
p(E)=C \exp \left[-\frac{\left(E-E_{o}\right)^{2}}{2 \sigma^{2}}\right],
$$

where $C=\left(2 \pi \sigma^{2}\right)^{-1 / 2}$. For large lattices $(L \rightarrow \infty)$, we have

$$
p(E) \rightarrow \delta\left(E-E_{0}\right)
$$

From Eq. (24), we obtain

$$
\begin{gathered}
<E>=\int_{-\infty}^{\infty} E p(E) d E=E_{o} \\
<(E-<E>)^{2}>=\int_{-\infty}^{\infty}\left(E-E_{0}\right)^{2} p(E) d E=\sigma^{2},
\end{gathered}
$$

and

$$
<(E-<E>)^{4}>=\int_{-\infty}^{\infty}\left(E-E_{0}\right)^{4} p(E) d E=3 \sigma^{4} .
$$

Inserting these expressions into Eq. (11), we have

$$
V_{E}(L)=1-\frac{3 \sigma^{4}}{3\left(\sigma^{2}\right)^{2}}=0,
$$

for all values of the parameter $\sigma$ (that is, independently of the size of the lattice used in the simulation). Using the limiting distribution, given by Eq. (25), we also have

$$
<E>\rightarrow \int_{-\infty}^{\infty} E \delta\left(E-E_{0}\right) d E=E_{o}
$$

and

$$
<(E-<E>)^{n}>\rightarrow \int_{-\infty}^{\infty}\left(E-E_{o}\right)^{n} \delta\left(E-E_{o}\right) d E=0
$$

for all $n$. Hence, $V_{E}(L) \rightarrow 0$, as we have already obtained.

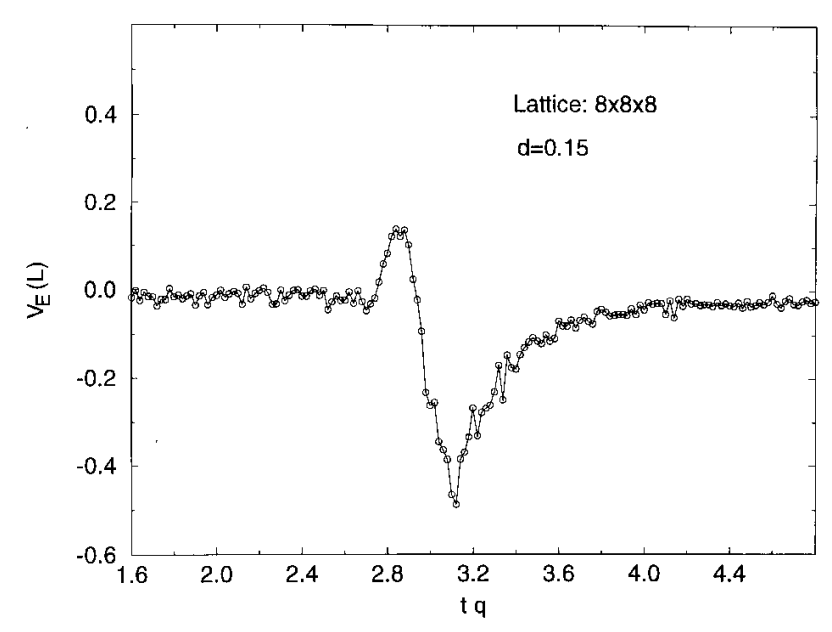

Figure 5. Fourth-order cumulant of energy versus temperature, $t q$, across a second-order phase transition. Monte Carlo data were obtained for the Blume-Capel model on a cubic lattice $(q=6)$ of side $L=8$, for $d=0.15$. Averages were calculated from 50000 Monte Carlo steps after thermalization. 
In a second-order transition the distribution $p(E)$ displays just a single peak, that moves from an initial value $E_{1}$ to a final value $E_{2}$. Therefore, the cumulant of energy across a second-order transition always vanishes, independently of the lattice size. The Monte Carlo estimates of $V_{E}(L)$ for the Blume-Capel model, as shown in Fig. (5), for $d=0.15$ and lattice size $L=8$, indicate a small maximum next to a small minimum near the second-order phase transition. This behavior suggests that it becomes too simple to describe the probabilities in the immediate neighborhood of a continuous transition by a symmetric Gaussian form. This is also hinted by the bottom left graph of Fig. (2), which is already quite asymmetric.

In a first-order phase transition there is a coexistence between the ordered phase, associated with a distribution of energy peaked at $E_{1}$, and the disordered phase, with a distribution peaked at $E_{2}$. Then, we can write

$$
p(E)=c \delta\left(E-E_{1}\right)+(1-c) \delta\left(E-E_{2}\right),
$$

from which we have

$$
\begin{gathered}
<E>=\int_{-\infty}^{\infty} E p(E) d E=c E_{1}+(1-c) E_{2} \\
<(E-<E>)^{2}>=\int_{-\infty}^{\infty}\left[E-c E_{1}-(1-c) E_{2}\right]^{2} p(E) d E=c(1-c)\left(E_{1}-E_{2}\right)^{2},
\end{gathered}
$$

and

$$
\begin{gathered}
<(E-<E>)^{4}>=\int_{-\infty}^{\infty}\left[E-c E_{1}-(1-c) E_{2}\right]^{4} p(E) d E= \\
=c(1-c)\left(1-3 c+3 c^{2}\right)\left(E_{1}-E_{2}\right)^{4} .
\end{gathered}
$$

The fourth-order cumulant is given by

$$
V_{E}(L)=2-\frac{1}{3 c(1-c)}
$$

which becomes negative for small values of either $c$ or $1-c$. In Fig. (6), for a cubic lattice of size $L=8$, at $t=0.15$, we show the fourth-order cumulant associated with the energy of the Blume-Capel model across a first-order phase transition.

In Figs. (7) and (8), we illustrate the fourthorder cumulants of magnetization and energy across a second-order transition. We see that the cumulants $V_{m}(L)$, for different values of $L$, cross at a unique point, which can be used to estimate the transition temperature[2, 6, 7, 13]. However, it should be pointed out that a precise location of the transition requires a detailed study of finite size scaling, which is beyond the scope of this paper[2, 3, 4, 6, 14, 15, 16].

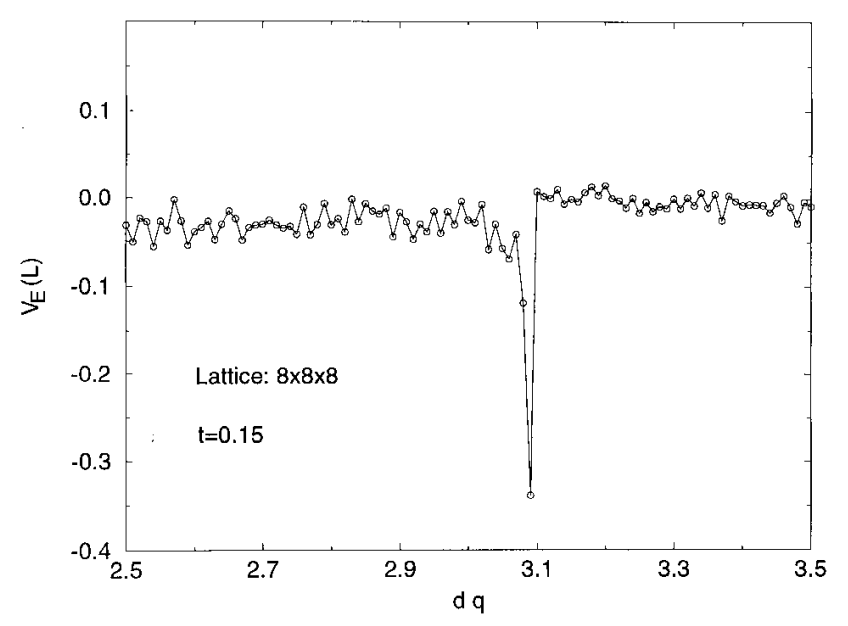

Figure 6. Fourth-order cumulant of energy versus the parameter of anisotropy, $d q$, across a first-order phase transition. Monte Carlo data were obtained for the Blume-Capel model on a cubic lattice $(q=6)$ of side $L=8$, at temperature $t=0.15$. Averages were calculated from 50000 Monte Carlo steps after thermalization. 


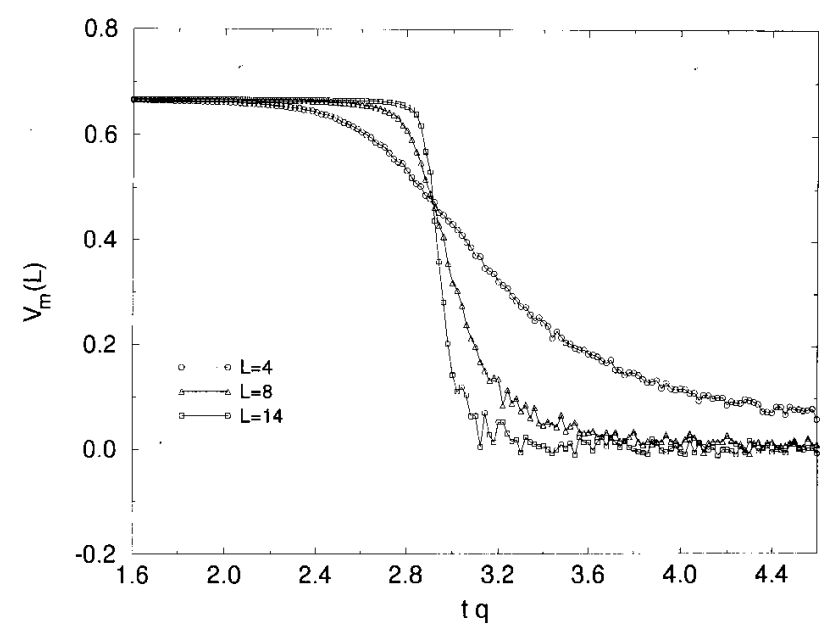

Figure 7. Fourth-order cumulant of magnetization versus temperature, $t q$, across a second-order phase transition $(d=0.15)$, for the Blume-Capel model on a sequence of cubic lattices $(q=6)$, of lattice sizes $L=4,8$, and 14 . The Monte Carlo averages were calculated from 50000 lattice steps after thermalization.

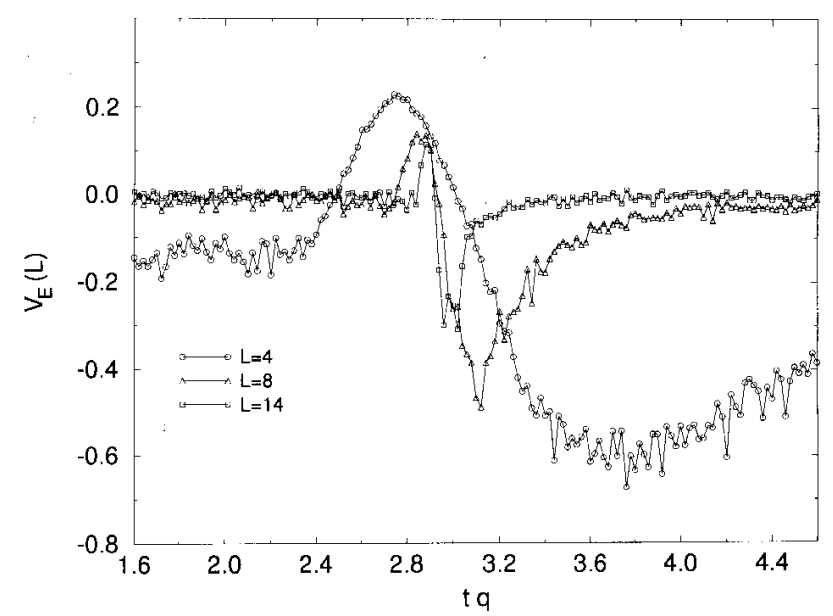

Figure 8. Fourth-order cumulant of energy versus temperature, $t q$, across a second-order phase transition $(d=0.15)$, for the Blume-Capel model on a sequence of cubic lattices $(q=6)$, of lattice sizes $L=4,8$, and 14. The Monte Carlo averages were calculated from 50000 lattice steps after thermalization.

Many authors use a fourth-order cumulant of the energy given by the form

$$
V_{E}(L)=1-\frac{<E^{4}>_{L}}{3<E^{2}>_{L}^{2}},
$$

instead of the connected expression of Eq. (11). Although this may work for Ising and Potts models[17], it is important to emphasize that for the Blume-Capel model we have to use the correct definition, given by Eq. (11), to be able to extract the order of the phase transition (see also the recent works of Janke[18], and of Borgs and collaborators[16]).

\section{Conclusions}

We have used the spin-1 Ising model of Blume and Capel to illustrate the feasibility of characterizing the order of a phase transition from a simple analysis of the behavior of the fourth-order cumulants of energy and magnetization. The general features of these cumulants can be derived from simple arguments of the theory of thermodynamic fluctuations. In the literature, there are two definitions of the fourth-order cumulant of energy. We have pointed out that, in the case of systems as the Blume-Capel model, it is important to consider the connected form of the cumulant of energy.

\section{Acknowledgments}

We acknowledge helpful discussions with C. E. I. Carneiro. This work was supported by grants from Fapesp and CNPq.

\section{References}

[1] K. Binder, Phys. Rev. Lett. 47, 693 (1981).

[2] K. Binder, Z. Phys. B43, 119 (1981).

[3] K. Binder and D. P. Landau, Phys. Rev. B30, 1477 (1984).

[4] M. S. S. Challa, D. P. Landau, and K. Binder, Phys. Rev. B34, 1841 (1986).

[5] A. Billoire, R. Lacaze, A. Morel, S. Gupta, A. Irback, and B. Petersson, Phys. Rev. B42, 6743 (1990).

[6] K. Vollmayr, J. D. Reger, M. Scheucher, and K. Binder, Z. Phys. B91,113 (1993).

[7] K. Binder and D. W. Heermann, Monte Carlo Simulations in Statistical Physics, Springer-Verlag, Berlin, 1988.

[8] N. Caticha, J. Chahine, and J. R. Drugowich de Felicio, Phys. Rev. B43 1173 (1991).

[9] M. Blume, V. J. Emery, and R. B. Griffiths, Phys. Rev. A4, 1071 (1971).

[10] M. N. Tamashiro and S. R. A. Salinas, Physica A211, 124 (1994).

[11] S. K. Ma, Statistical Mechanics, World Scientific, Singapore, 1985.

[12] J. Oitmaa, J. F. Fernandez, Phys. Rev. B39, 11920 (1989).

[13] M. Plischke and B. Bergersen, Equilibrium Statistical Physics, World Scientific, Singapore, 1994. 
[14] K. Binder, M. Nauenberg, V. Privman, and A. P. Young, Phys. Rev. B31, 1498 (1985).

[15] J. Lee and J. M. Kosterlitz, Phys. Rev. B43, 3265 (1991).

[16] C. Borgs, P. E. L. Rakow and S. Kappler, J. Phys. I
France 4, 1027 (1994).

[17] J. F. McCarthy, Phys. Rev. B41, 9530 (1990).

[18] W. Janke, Phys. Rev. B 47, 14757 (1993). 